\title{
Avaliação do Desenvolvimento Motor de Crianças Nascidas Prematuras: Impacto de Fatores de Risco Maternos
}

\author{
Assessment of Motor Development in Children Born prematurely: Impact of Maternal Risk Factors
}

\author{
Fernanda Trubian ${ }^{1}$ \\ Mayra Nathu Lodi ${ }^{1}$ \\ Caroline Cenci Sangali ${ }^{1}$ \\ Laura de Moura Rodrigues ${ }^{2}$ \\ Raquel Saccani ${ }^{3}$
}

\section{RESUMO}

Objetivo: Avaliar o impacto dos fatores de risco maternos sobre o desenvolvimento motor infantil de bebês nascidos prematuros, de 0 a 12 meses de idade corrigida. Metodologia: Participaram 74 bebês nascidos prematuros, cadastrados no Ambulatório do Centro Clínico da Universidade de Caxias do Sul. Para avaliação do desenvolvimento motor dos bebês foi utilizada a Alberta Infant Motor Scale, além de dois questionários para controle de variáveis referentes a fatores de risco biológicos e ambientais. Foi utilizada estatística descritiva e o teste $\mathrm{Chi}^{2} \mathrm{de}$ Pearson $(p=0,05)$. Resultados: A categorização de desempenho geral da amostra indicou que $14,9 \%$ das crianças foram classificadas com atraso para o desenvolvimento motor e $29,7 \%$ com suspeita de atraso. Ao associar o desempenho motor com os fatores de risco maternos, destaca-se que as crianças que receberam aleitamento materno apresentaram melhor desempenho motor $\left(\mathrm{Chi}^{2}=3,69 ; \mathrm{p}=0,05\right)$, onde apenas $37,1 \%$ $(n=13)$ da amostra estava abaixo do esperado. Também foi observada associação significativa entre o grau de escolaridade da mãe do bebê e o desempenho motor $\left(\mathrm{Chi}^{2}=5,27 ; \mathrm{p}=0,02\right)$. Conclusão: Os achados deste estudo indicam que os fatores maternos podem impactar o desenvolvimento motor de bebês nascidos prematuros. A partir dos resultados encontrados, reforça-se a importância da intervenção, que deve ser iniciada precocemente, a fim de reverter ou minimizar atrasos motores.

\section{DESCRITORES}

Recém-nascido Prematuro. Desenvolvimento Infantil. Fatores de Risco.

\begin{abstract}
Objective: To analyze the impact of maternal risk factors on the motor development of infants born prematurely, from 0 to 12 months of corrected age. Methodology: A total of 74 babies born prematurely ( $<36$ weeks), participated in the research, registered at the Ambulatório Central da Universidade de Caxias do Sul. To assess the motor development of the babies, the Alberta Infant Motor Scale was used, in addition to two questionnaires to control variables related to biological and environmental risk factors. Descriptive statistics and Pearson's $\mathrm{Chi}^{2}$ test $(p=0,05)$ were used. Results: The general performance categorization of the sample indicated that $14.9 \%$ of children were classified as delayed in motor development and $29.7 \%$ with suspected delay. When associating motor performance with maternal risk factors, children who received breastfeeding showed better motor performance $\left(\mathrm{Chi}^{2}=3.69 ; \mathrm{p}=0.05\right)$, whereas only $37.1 \%(n=13)$ of the sample was below expected. There was also a significant association between the baby's mother's level of education and motor performance $\left(\mathrm{Chi}^{2}=5.27\right.$; $p=0.02)$. Conclusions: The findings of this study indicate that maternal factors can impact the motor development of babies born prematurely. Based on the results found, the importance of the intervention is reinforced, which must be started early, in order to reverse or minimize motor delays.
\end{abstract}

\section{DESCRIPTORS}

Premature Infant. Child Development. Risk Factors.

${ }^{1}$ Fisioterapeuta, graduada pela Universidade de Caxias do Sul, Caxias do Sul, Rio Grande do Sul, Brasil.

2 Fisioterapeuta. Mestranda em Ciências da Saúde e Ciências da Vida pela Universidade de Caxias do Sul, Caxias do Sul, Rio Grande do Sul, Brasil.

${ }^{3}$ Pós-Doutora em Ciências do Movimento Humano pela Universidade Federal do Rio Grande do Sul e Docente do Curso de Fisioterapia da Universidade de Caxias do Sul, Caxias do Sul, Rio Grande do Sul, Brasil. 
A primeira infância diz respeito a um período específico do desenvolvimento onde ocorre a maior maturação cerebral, sendo esta fase determinante para a evolução do bebê. Em países subdesenvolvidos, as crianças têm maior dificuldade de atingir seu potencial de desenvolvimento devido a uma maior exposição à riscos biológicos, psicossociais e ambientais ${ }^{1}$. O nascimento prematuro, que tem como definição qualquer nascimento que acontece antes de 37 semanas de gestação, é considerado como a principal causa de mortes neonatais nestes países, além de contribuir de forma significativa para morbidades infantis ${ }^{2}$. A vulnerabilidade característica do prematuro afeta seu desenvolvimento neuropsicomotor, podendo ser intensificado por desvantagens ambientais e repercutir ao longo da vida da criança ${ }^{3}$.

Os bebês prematuros apresentam trajetória de aquisições motoras diferente das crianças nascidas a termo, sendo descritas inferioridades no desempenho destes, principalmente no primeiro ano de vida $^{1,3}$, uma vez que, a prematuridade está entre os fatores determinantes para alterações motoras, onde os atrasos se tornam maiores quanto menor for a idade gestacional ${ }^{4,5}$. Além disso, já está determinado que os bebês prematuros podem ter suas aquisições motoras potencializadas ou inibidas por fatores relacionados tanto ao seu ambiente de inserção, físico, social e cultural ${ }^{6,7}$, quanto a fatores relacionados à mãe, como aleitamento materno e o nível de educação ${ }^{8}$.

Entretanto, fatores associados à saúde materna, como a hipertensão, o diabetes e a pré-eclâmpsia, embora menos investigados, estão associados à uma baixa pontuação na Escala de Apgar (aparência, pulso, gesticulação, atividade e respiração), sendo este um método que avalia o prognóstico da saúde neurológica e desenvolvimento cognitivo futuro de recém-nascidos ${ }^{9}$. Por isso, fatores relacionados à saúde materna ainda representam uma importante lacuna a ser investigada, considerando sua possível interferência no desenvolvimento infantil.

Diante do exposto, a avaliação do desenvolvimento motor de prematuros e sua associação com fatores de risco maternos se faz essencial para a identificação de déficits, de forma que a intervenção possa ser iniciada precocemente, podendo não só reverter os atrasos identificados, como também, potencializar as aquisições que a criança já apresenta, estimulando, assim, habilidades e prevenindo a perda da funcionalidade ${ }^{1,5,10}$. Sendo assim, o objetivo deste estudo foi avaliar o impacto dos fatores de risco maternos sobre o desenvolvimento motor de bebês nascidos prematuros, de 0 a 12 meses de idade corrigida, cadastrados no Ambulatório de Alto Risco do Centro Clínico da Universidade de Caxias do Sul (UCS).

\section{METODOLOGIA}

\section{Delineamento}

Pesquisa descritiva e observacional, de caráter associativo, com abordagem transversal, aprovada pelo Comitê de Ética em Pesquisa da UCS (Parecer $n^{\circ}$. 2.688.022).

\section{Amostra}

A amostra foi composta por 74 bebês 
nascidos prematuros ( $<37$ semanas), com idade corrigida de 0 a 12 meses, cadastradas no Ambulatório de Alto Risco do Centro Clínico da UCS. Este Ambulatório é uma unidade do Sistema Único de Saúde (SUS) e atende a 49 municípios da Região da Serra Gaúcha. As coletas foram realizadas entre maio de 2018 a julho de 2019.

Os bebês que fizeram parte do estudo foram incluídos com a autorização dos pais, obedecendo aos seguintes critérios: a) ser encaminhado para o Ambulatório de Alto Risco do Centro Clínico da UCS, após período de internação na UTI Neonatal do Hospital Geral de Caxias do Sul; b) nascidos prematuros (36 semanas de gestação ou menos); c) estar dentro da faixa etária de 0 a 12 meses, considerando a idade corrigida; d) estar em acompanhamento pelos profissionais de Fisioterapia no Ambulatório de Alto Risco. Como critérios de exclusão, considerou-se: a) impossibilidade de completar a avaliação; b) diagnóstico confirmado de doenças neurológicas; c) idade gestacional superior a 36 semanas; d) alteração de sinais vitais, sono profundo e choro intenso, por se tratarem de condições que podem interferir no desempenho das crianças; e) não consentimento dos pais. Sendo assim, excluiu-se 32 bebês, sendo 18 com doenças neurológicas, 12 nascidos a termo e dois bebês que não completaram a avaliação.

\section{Instrumentos e Procedimentos}

Para avaliação do desenvolvimento motor dos bebês foi utilizada a Alberta Infant Motor Scale (AIMS), desenvolvida por Piper e Darrah em 1992 no Canadá, e validada no
Brasil ${ }^{11}$. Trata-se de uma escala observacional, dividida nas posturas de prono (21 itens), supino (9 itens), sentado (12 itens) e em pé (16 itens), que representam a sequência do desenvolvimento motor e conta com descritores em cada posição avaliada. Para cada item alcançado pela criança, pontuase um ponto, enquanto para as posturas ainda não adquiridas, pontua-se zero. Ao final, somam-se os itens avaliados em cada postura, sendo que o total pode variar de 0 a 58 pontos. Após, este escore é convertido em um percentual, de 0 a $100 \%$, onde se considera: valores abaixo de $5 \%$ indicam desenvolvimento motor anormal, valores entre $6 \%$ e $25 \%$ indicam desenvolvimento com suspeita para atraso motor, e valores acima de $25 \%$ indicam desenvolvimento motor dentro da normalidade ${ }^{11}$.

Ainda, foi aplicado um questionário simples para identificar as características biológicas e ambientais da amostra. Este instrumento contava com itens relacionados à criança e a saúde materna, tais como: idade cronológica e corrigida, idade gestacional, idade ao nascer, comorbidades da mãe, idade dos pais, Apgar e tempo de internação hospitalar.

Ao chegarem ao Ambulatório de Alto Risco, acompanhadas por seus pais ou responsáveis, os bebês eram recebidos pela equipe multiprofissional, composta por diversas especialidades, entre estas, a Equipe de Fisioterapia. Em uma sala específica, primeiramente, explicou-se aos pais o processo de avaliação da criança e, após a assinatura do termo de consentimento livre e esclarecido, foram aplicados os questionários da pesquisa, além da coleta de dados por 
meio da Carteira Nacional de Vacinação e do prontuário de controle do Ambulatório.

Após, foi realizada a avaliação motora com a AIMS, com duração média de 20 minutos, sendo que as pesquisadoras tinham sido treinadas previamente com o instrumento. Durante a avaliação, as crianças foram estimuladas por meio de brinquedos sonoros e coloridos, nas quatro posturas da escala, com o mínimo de manuseio pelos terapeutas e com o mínimo de roupas possíveis. A escala preconiza que, observar o bebê ao invés de manuseá-lo permite que o mesmo mostre suas habilidades, já que o manuseio pode induzir o bebê a demonstrar reações específicas.

Para análise dos dados, utilizouse o programa estatístico SPSS (versão 20.0). Foi utilizada análise descritiva, com distribuição de frequência simples, medidas de tendência central (média) e de variabilidade (desvio padrão). Para as associações entre características e categorização do desenvolvimento motor, foi utilizado o teste $\mathrm{Chi}^{2}$ de Pearson, com nível de significância de $5 \%$.

\section{RESULTADOS}

As características biológicas gerais estão dispostas na Tabela 1, onde se observou, por meio da média de idade gestacional, que existiu uma prevalência de prematuridade moderada. Além disso, grande parte dos bebês nasceu com o peso inferior a $1500 \mathrm{~g}$, caracterizados com muito baixo peso.

No que se refere às características

Tabela 1. Características biológicas (média, desvio padrão, mínimo e máximo) da amostra

\begin{tabular}{|c|c|c|c|c|}
\hline Características Biológicas & Média & DP & Minı & Máx \\
\hline Idade gestacional (semanas) & 31 & 2,48 & 25 & 36 \\
\hline Idade corrigida (meses) & 3,42 & 3,14 & - & 11 \\
\hline Idade cronológica (meses) & 5,51 & 3,14 & 1 & 12 \\
\hline Peso ao nascer $(\mathrm{g})$ & 1378,23 & 433,71 & 600 & 2960 \\
\hline Comprimento ao nascer $(\mathrm{cm})$ & 38,69 & 3,80 & 27 & 48 \\
\hline Perímetro cefálico $(\mathrm{cm})$ & 28,09 & 2,49 & 22 & 34 \\
\hline $\mathrm{N}^{\circ}$ de Consultas pré-natal & 5,66 & 2,10 & - & 10 \\
\hline Internação hospitalar (dias) & 51,36 & 25,62 & 24 & 137 \\
\hline Uso de VM (dias) & 10,40 & 15,75 & - & 74 \\
\hline Apgar $5^{\circ}$ minuto & 8,29 & 0,97 & 5 & 9 \\
\hline Peso Atual $(\mathrm{g})$ & 5364,38 & 1814,34 & 2650 & 10600 \\
\hline Comprimento Atual (cm) & 57,95 & 7,86 & 46 & 79 \\
\hline & FR & \multicolumn{3}{|c|}{$\%$} \\
\hline \multicolumn{5}{|l|}{ Sexo } \\
\hline Feminino & \multirow{2}{*}{\multicolumn{2}{|c|}{$\begin{array}{l}35 \\
39\end{array}$}} & & \\
\hline Masculino & & & \multicolumn{2}{|c|}{52,7} \\
\hline \multicolumn{5}{|l|}{ Classificação Prematuridade } \\
\hline Limítrofe & \multicolumn{2}{|l|}{8} & \multicolumn{2}{|c|}{10,8} \\
\hline Moderado & 39 & & \multicolumn{2}{|c|}{52,7} \\
\hline Extremo & \multicolumn{2}{|l|}{27} & \multicolumn{2}{|c|}{36,5} \\
\hline \multicolumn{5}{|l|}{ Classificação Peso } \\
\hline Normal & 2 & & \multicolumn{2}{|c|}{2,7} \\
\hline Baixo Peso & 21 & & \multicolumn{2}{|c|}{28,4} \\
\hline Muito Baixo Peso & 42 & & \multicolumn{2}{|c|}{56,8} \\
\hline Extremo Baixo Peso & 9 & & \multicolumn{2}{|c|}{12,2} \\
\hline
\end{tabular}


maternas e ambientais, dispostas na Tabela 2 , destaca-se que $52,7 \%$ dos bebês não receberam aleitamento materno e 91,9\% não receberam aleitamento exclusivo. Além disso, a renda familiar até dois salários mínimos foi a mais frequente, assim como a escolaridade dos pais até ensino médio incompleto. Cabe destacar que a idade dos pais variou de 14 a 52 anos, com média materna de $28,24 \pm 7,53$ anos e paterna de $21,60 \pm 8,84$ anos. Ainda, a média de filhos foi de 2,06 $\pm 1,37$ anos.

A categorização de desempenho geral, disposta na Figura 1, demonstrou que $44,6 \%$ dos bebês foram classificados com desenvolvimento abaixo do esperado para a idade. Ainda considerando o desempenho motor, as crianças apresentaram percentil médio de $37,18 \pm 26,67$, indicando que, embora grande parte tenha sido classificada como normalidade para o desenvolvimento, a média obtida ficou abaixo de 50 .

Ao associar o desempenho motor com os fatores de risco maternos, observou-se que os bebês que receberam aleitamento materno apresentaram melhor desempenho motor, onde apenas $37,1 \%(n=13)$ da amostra estava abaixo do esperado $\left(\mathrm{Chi}^{2}=3,69 ; \mathrm{p}=0,05\right)$. Também foi observada associação significativa

Tabela 2. Características ambientais e maternas da amostra

\begin{tabular}{|c|c|c|}
\hline Características Ambientais e Maternas & FR & $\%$ \\
\hline \multicolumn{3}{|l|}{ Renda em salários } \\
\hline 1 a 2 & 40 & 4,1 \\
\hline 3 a 4 & 22 & 29,7 \\
\hline 5 a 7 & 04 & 5,4 \\
\hline Não respondeu & 07 & 9,5 \\
\hline Sem renda fixa & 01 & 1,4 \\
\hline \multicolumn{3}{|l|}{ Escolaridade do pai } \\
\hline Nunca estudou & 01 & 1,4 \\
\hline $1^{\circ}$ arau incompleto & 21 & 28.4 \\
\hline $1^{\circ} \mathrm{grau}$ completo & 11 & 14,9 \\
\hline Ensino médio incompleto & 11 & 14,9 \\
\hline Ensino médio completo & 19 & 25,7 \\
\hline Ensino superior & 05 & 6,8 \\
\hline Não respondeu & 06 & 8,1 \\
\hline \multicolumn{3}{|l|}{ Escolaridade da mãe } \\
\hline Nunca estudou & - & - \\
\hline $1^{\circ}$ grau incompleto & 15 & 20,3 \\
\hline $1^{\circ}$ grau completo & 15 & 20,3 \\
\hline Ensino médio incompleto & 14 & 18,9 \\
\hline Ensino médio completo & 18 & 24,3 \\
\hline Ensino superior & 06 & 8,1 \\
\hline Não respondeu & 06 & 8,1 \\
\hline \multicolumn{3}{|l|}{ Hipertensão pré-gestacional } \\
\hline Sim & 14 & 18.9 \\
\hline Não & 60 & 81,1 \\
\hline \multicolumn{3}{|l|}{ Hipertensão gestacional } \\
\hline Sim & 28 & 37,8 \\
\hline Não & 46 & 62.2 \\
\hline \multicolumn{3}{|l|}{ Diabetes gestacional } \\
\hline Sim & 10 & 13,5 \\
\hline Não & 64 & 86,5 \\
\hline \multicolumn{3}{|l|}{ Síndrome de Helln } \\
\hline Sim & 08 & 10,8 \\
\hline Não & 66 & 89.2 \\
\hline
\end{tabular}


Figura 1. Distribuição (\%) da classificação obtida na AIMS pelos bebês da amostra

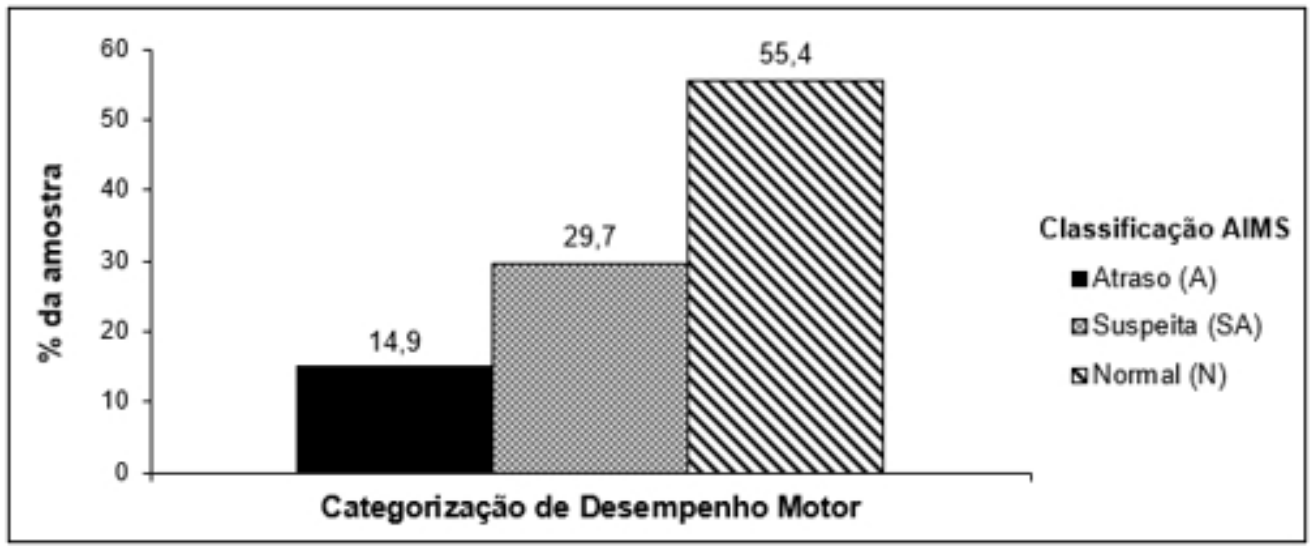

entre o grau de escolaridade da mãe do bebê e o desempenho motor $\left(\mathrm{Chi}^{2}=5,27 ; \mathrm{p}=0,02\right)$. A análise detalhada das associações entre a categorização de desempenho e características maternas está disposta na Tabela 3.

Embora as variáveis hipertensão prégestacional e o diabetes gestacional não tenham demonstrado associação significativa em relação ao desempenho, observouse que o maior percentil de atraso para o desenvolvimento motor correspondeu aos bebês cujas mães apresentaram ambas condições. Constatou-se que $28,6 \%$ dos filhos de mães hipertensas foram classificadas com atraso motor, assim como aqueles em que as mães desenvolveram diabetes gestacional, onde $20 \%$ foram classificados da mesma forma.

\section{DISCUSSÃO}

O presente estudo realizou a análise do impacto do risco de fatores maternos sobre o desenvolvimento motor de bebês nascidos prematuros, até 12 meses de idade, indicando que o desempenho motor dos avaliados pode ter sido influenciado por esses fatores.

Os resultados demonstraram que parte dos bebês apresentou desenvolvimento motor inadequado para a idade. O desempenho motor abaixo do esperado pode ser justificado por diferentes fatores de risco, aos quais

Tabela 3. Associações entre a categorização do desempenho motor e fatores maternos

\begin{tabular}{l|c|c}
\hline Desempenho motor X Fatores de Risco & Chi $^{\mathbf{2}}$ & $\mathrm{p}$ \\
\hline Quantidade de filhos & 0,115 & 0,73 \\
Escolaridade da mãe & 5,273 & $0,02^{*}$ \\
Amamentação & 3,698 & $0,05^{\mathbf{2}}$ \\
Hipertensão pré gestacional & 2,342 & 0,12 \\
Hipertensão gestacional & 1,572 & 0,21 \\
Síndrome de Hellp & 0,151 & 0,69 \\
Diabetes Gestacional & 0,838 & 0,36 \\
\hline
\end{tabular}

Legenda: $\mathrm{Chi}^{2}=$ Teste Qui quadrado de Pearson; * =significativo $\mathrm{p} \leq 0,05$ 
os bebês, principalmente os prematuros, são expostos. Os bebês que nascem prematuramente apresentam um risco maior para comprometimentos motores e cognitivos, em relação aos nascidos a termo. Fatores como o peso ao nascer, o período de hospitalização e o tempo de ventilação mecânica são associados a atrasos no desenvolvimento motor ${ }^{12}$.

À medida que o peso ao nascer e a idade gestacional diminuem, aumenta a associação às anormalidades do neurodesenvolvimento, principalmente para bebês nascidos com menos de 32 semanas, sendo que os comprometimentos neurológicos podem persistir durante a adolescência e a vida adulta ${ }^{10,13}$. Desta forma, os prematuros nascidos com baixo peso e muito baixo peso, ou seja, abaixo de $2500 \mathrm{~g} \mathrm{e}$ $1500 \mathrm{~g}$, respectivamente, estão em um grupo considerado de alto risco para distúrbios motores durante os primeiros anos de vida ${ }^{14}$. Considerando os participantes deste estudo, essas características ajudam a explicar os resultados de desempenho observados.

Atrasos motores têm sido associados não somente à saúde infantil. Fatores maternos e ambientais, como o nível de escolaridade e renda, comorbidades da mãe pré-gestacionais e durante a gravidez, pouca interação social, número de filhos, falta de oferta de aleitamento materno e de oportunidades motoras do ambiente em que a criança está inserida, são considerados riscos em potencial para atrasos no desenvolvimento motor, cognitivo e social ${ }^{8,15}$.

Neste estudo, a relação entre a escolaridade materna e o desenvolvimento se mostrou significativa, onde os bebês nascidos de mães com maior escolaridade obtiveram melhores índices na AIMS. A literatura relata que o nível escolar materno tem um impacto significativo no neurodesenvolvimento de prematuros, sugerindo que este seja o fator de maior impacto em relação aos demais fatores socioeconômicos. O baixo nível educacional pode estar relacionado a pouca interação entre adulto e criança, além de menores oportunidades motoras, influenciando, além do desempenho motor, o desenvolvimento da linguagem da criança ${ }^{16,17}$. De forma semelhante, Wang et al. ${ }^{18}$, em estudo prévio, relataram que o nível de escolaridade da mãe teve influência sobre o controle postural de bebês pré-termos avaliados até os 12 meses de idade pela AIMS. Corroborando com estes resultados, autores afirmam que a escolaridade materna, abaixo do nível ensino médio, está associada a fatores cognitivos e atrasos na linguagem; e níveis educacionais mais altos, se tornam um fator de proteção para o desenvolvimento destas habilidades ${ }^{19}$.

Os resultados obtidos na AIMS demonstraram que ocorreu uma associação significativa entre o desempenho motor dos bebês e o aleitamento materno, indicando que quanto maior o tempo de amamentação, melhor o desenvolvimento. A amamentação é benéfica à saúde infantil por fornecer nutrientes e energia, além de hormônios, anticorpos e fatores de crescimento, que auxiliam no desenvolvimento dos bebês. Além disso, mães que amamentam apresentam uma maior sensibilidade materna, que se refere à capacidade da mãe de ler e responder adequadamente os sinais da criança e que está associado a um melhor desenvolvimento neurológico dos lactentes ${ }^{20-22}$. 
Alinhando-se a esta pesquisa, um estudo prévio com prematuros observou que as crianças amamentadas por um tempo menor ou igual há dois meses, obtiveram menores escores do que aquelas amamentadas por mais de três meses. Estas apresentaram valores mais altos para os domínios motor e de linguagem ${ }^{23}$. Sob o mesmo aspecto, outros autores também relataram que o aleitamento materno foi associado ao desenvolvimento motor, onde escores superiores são observados em bebês que foram aleitados por mais tempo, sugerindo um período mínimo de amamentação de seis meses para prevenir atrasos motores. Pesquisas longitudinais acrescentam que a amamentação pode ter influência em longo prazo no desenvolvimento motor, onde bebês que receberam aleitamento materno por mais de seis meses apresentam melhor desempenho motor durante a adolescência ${ }^{24,25}$.

Poucos são os estudos que descrevem a interferência das condições de saúde da mãe sobre as aquisições motoras e cognitivas da criança ${ }^{26,27}$. Entretanto, ainda que não tenha associação significativa, observou-se, neste estudo, que as variáveis maternas: diabetes gestacional e hipertensão pré-gestacional, podem ter interferido no desempenho motor, pois, o maior percentil de atraso no desenvolvimento corresponde às crianças cujas mães apresentam ambas condições. O diabetes gestacional pode ter uma série de consequências para as mães e para os bebês, dentre elas, abortos espontâneos, malformações congênitas, comprometimentos motores e de linguagem, além de déficits de atenção e transtornos de hiperatividade ${ }^{26}$. De encontro aos achados do presente estudo, outros autores, ao avaliarem o desenvolvimento motor de bebês em diferentes etapas até os 24 meses, relataram que aqueles nascidos de mães que desenvolveram diabetes na gestação demoraram mais tempo para sentar sem apoio, caminhar com apoio e caminhar de forma independente ${ }^{27}$.

Os distúrbios hipertensivos da gestação incluem a hipertensão pré-gestacional, considerada crônica, além da hipertensão gestacional e a pré-eclâmpsia, sendo que a prematuridade é duas vezes mais comum em bebês de mulheres que apresentam algumas destas condições. Além disso, estas crianças apresentam maior risco para comprometimentos no desenvolvimento motor, da linguagem e disfunções ${ }^{28,29}$. Neste contexto, um estudo que avaliou bebês nascidos de mães com distúrbios hipertensivos, não encontrou associação significativa em relação ao desenvolvimento motor, porém, observou que estas crianças foram classificadas como pequenas para idade gestacional (PIG). O estudo sugere que o crescimento gestacional abaixo do ideal em prematuros pode ter impacto sobre o desenvolvimento motor, sendo necessária a intervenção para estes bebês, devido à vulnerabilidade apresentada ${ }^{28}$.

A partir dos resultados encontrados, reforça-se a importância da intervenção, que deve ser iniciada precocemente, a fim de reverter ou minimizar atrasos motores. A intervenção precoce, logo após a alta hospitalar, se concentra em fatores familiares e ambiente doméstico, sendo que as estratégias são voltadas para minimizar os efeitos da prematuridade, promovendo o desempenho 
motor $^{30}$. Desta forma, as orientações aos pais e cuidadores são importantes por possibilitar cuidados e estímulos adequados, que podem ser realizados diariamente, aumentando também a interação entre pais e bebês, contribuindo para a melhora do desenvolvimento ${ }^{1}$.

\section{CONCLUSÃO}

Embora estudos transversais limitem a investigação do processo de aquisições motoras e de sua variabilidade, os resultados encontrados neste estudo indicaram que fatores de risco maternos podem impactar o desenvolvimento motor de bebês nascidos prematuros. Ainda, sugere-se um acompanhamento longitudinal e ampliação da amostra, para que seja possível avaliar o impacto dos fatores de risco maternos ao longo do desenvolvimento destes bebês.

\section{REFERÊNCIAS}

1. Ferreira RC, Alves CRL, Guimarães MAP, Menezes KKP, Magalhães LC. Effects of early interventions focused on the family in the development of children born preterm and/or at social risk: a meta-analysis. J Pediatr (Rio J). 2020; 96(1):20-38

2. Quinn J, Munoz FM, Gonik B, Frau L, Cutland C Mallett-Moore T, Kissou A, Wittke F, Das M, Nunes T, Pye S, Watson W, Ramos AA, Cordero JF, Huang W, Kochhar S, Buttery J, The Brighton Collaboration Preterm Birth Working Group. Preterm birth: Case definition \& guidelines for data collection, analysis,and presentation of immunisation safety data. Vaccine. 2016; 34(49):60476056

3. Yaari M, Mankuta D, Harel-Gadassi A, Friedlander E, BarOz B, Eventov-Friedman S, Maniv N, Zucker D, Yirmiya N. Early developmental trajectories of preterm infants. Res Dev Disabil. 2018; 81:12-23.

4. Sampaio TF, Nogueira KPA, Pontes TB, Toledo AM Comportamento motor de lactentes prematuros de baixo peso e muito baixo peso ao nascer. Fisioter Pesqui. 2015 22(3):253-260

5. Albuquerque PL, Guerra MQF, Lima MC, Eickmann SH Concurrent validity of the Alberta Infant Motor Scale to detect delayed gross motor development in preterm infants: A comparative study with the Bayley III. Dev Neurorehabil. 2017; 21(6):408-414

6. Stelmach I, Kwarta P, Jerzynska J, Stelmach W, Krakowiak J, Karbownik M, Podlecka D, Hanke W, Polanska K. Duration of breastfeeding and psychomotor development in 1-year-old children - Polish Mother and Child Cohort Study. Int J Occup Med Environ Health. 2019; 32(2):175184.

7. Zago JTC, Pinto PAF, Leite HR, Santos JN, Morais RLS Associação entre o desenvolvimento neuropsicomotor e

fatores de risco biológico e ambientais em crianças na primeira infância. 2017; 19(3):320-329.

8. Pereira KRG, Valentini NC, Saccani R. Brazilian infant motor and cognitive development: Longitudinal influence of risk factors. Pediatr Int. 2016; 58(12):1297-1306

9. Almeida NKO, Pedreira CE, Almeida RMVR. Impact of maternal education level on risk of low Apgar score. Public Health. 2016; 140:244-249.

10. Fuentefria RN, Silveira RC, Procianoy RS. Motor development of preterm infants assessed by the Alberta Infant Motor Scale: systematic review article. J Pediatr (Rio J). 2017; 93(4):328-342

11. Valentini NC, Saccani R. Brazilian validation of the Alberta Infant Motor Scale. Phys Ther. 2012; 92(3), 440-447.

12. Miyagishima S, Asaka T, Kamatsuka K, Kozuka N, Kobayashi M, Igarashi L, Hori T, Tsutsumi H. Spontaneous movements of preterm infants is associated with outcome of gross motor development. Brain Dev. 2018; 40(8):627633.

13. Olsen JE, Allinson LG, Doyle LW, Brown NC, Lee KJ, Eeles AL, Cheong JLY, Spittle AJ. Preterm and term-equivalent age general movements and 1-year neurodevelopmental outcomes for infants born before 30 weeks' gestation. Dev Med Child Neurol. 2018; 60(1):47-53.

14. Su YH, Jeng SF, Hsieh WS, Tu YK, Wu YT, Chen LC. Gross motor trajectories during the first year of life for preterm infants with very low birth weight. Phys Ther. 2017; 97(3):365-73.

15. Namazzi G, Hildenwall H, Mubiri P, Hanson C, Nalwadda C, Nampijja M, Kakooza-Mwesige A, Waiswa P, Tumwine J. Prevalence and associated factors of neurodevelopmental disability among infants in eastern Uganda: a population 
based study. BMC pediatr (Online). 2019; 19(1):379-389.

16. Patra K, Greene MM, Patel AL, Meier P. Maternal education level predicts cognitive, language, and motor outcome in preterm infants in the second year of life. Am J Perinatol. 2016; 33(8):738-744.

17. Zerbeto AB, Cortelo FM, Filho EBC. Association between gestational age and birth weight on the language development of Brazilian children: a systematic review. J Pediatr (Rio J). 2015; 91(4):326-332.

18. Wang TN, Howe TH, Hinoiosa J, Hsu YW. Postural control of pre-term infants at 6 and 12 months corrected age. Early Hum Dev. 2010; 86(7);433-437.

19. Agarwal PK, Shi L, Rajadurai VS, Zheng Q, Yang $\mathrm{PH}$, Khoo PC, Quek BH, Daniel LM. Factors affecting neurodevelopmental outcome at 2 years in very preterm infants below 1250 grams: a prospective study. J Perinatol. 2018; 38(8):1093-1100.

20. Michels KA, Ghassabian A, Mumford SL, Sundaram R, Bell EM, Bello SC, Yeung EH. Breastfeeding and motor development in term and preterm infants in a longitudinal US cohort. Am J Clin Nutr. 2017; 106(6):1456-1462.

21. Belfort MB, Anderson PJ, Nowak VA, Lee KJ, Molesworth C, Thompson DK, Doyle LW, Inder TE. Breast Milk Feeding, Brain Development, and Neurocognitive Outcomes: A 7-Year Longitudinal Study in Infants Born at Less Than 30 Weeks' Gestation. J Pediatr. 2016; 177:133139.

22. Firk C, Konrad K, Herperts-Dahlamnn B, Scharke W, Dahmen B. Cognitive development in children of adolescent mothers: The impact of socioeconomic risk and maternal sensitivity. Infant Behav Dev. 2018; 50:238-246.

23. Eickmann SH, Malkes NFA, Lima MC. Psychomotor development of preterm infants aged 6 to 12 months. São Paulo Med J. 2012;130(5):299-306.
24. Pereira KRG, Saccani R, Valentini NC. Cognição e ambiente são preditores do desenvolvimento motor de bebês ao longo do tempo. Fisioter Pesqui (Online). 2016; 23(1):59-67.

25. Grace T, Oddy W, Bulsara M, Hands B. Breastfeeding and motor development: A longitudinal cohort study. Hum Mov Sci. 2017; 51:9-16.

26. Márquez-Valadez B, Valle-Bautista R, García-Lopez G, Díaz NF, Molina-Hernández A. Maternal Diabetes and Fetal Programming Toward Neurological Diseases: Beyond Neural Tube Defects. Front Endocrinol. 2018; 9:738-744.

27. Ghassabian A, Sundaram R, Wylie A, Bell E, Bello S, Yeung $E$. Maternal medical conditions during pregnancy and gross motor development up to age 24 months in the Upstate KIDS study. Dev Med Child Neurol. 2015; 58(7):728-734.

28. Avorgbedor F, Silva S, Merwin E, Blumenthal JA Holditch-Davis D. Health, Physical Growth, and Neurodevelopmental Outcomes in Preterm Infants of Women With Hypertensive Disorders of Pregnancy. J Obstet Gynecol Neonatal Nurs. 2019; 48(1):69-77.

29. Grace T, Oddy W, Bulsara M, Hands B. Breastfeeding and motor development: A longitudinal cohort study. Hum Mov Sci. 2017; 51:9-516.

30. Spittle A, Orton J, Anderson PJ, Boyd R, Doyle LW. Early developmental intervention programmes provided post hospital discharge to prevent motor and cognitive impairment in preterm infants. Cochrane database syst rev (Online). 2015; (11):1-25.

\section{CORRESPONDÊNCIA}

Raquel Saccani

Rua Francisco Getúlio Vargas, 1130

Bairro Petrópolis - Caxias do Sul - RS, Brasil.

E-mail: rsaccani@ucs.br 